\title{
A Hybrid Analytical-Numerical Method for Solving Advection-Dispersion Problems on a Half-Line
}

\author{
F.P.J. de Barros ${ }^{\mathrm{a}, *}$, M.J. Colbrook ${ }^{\mathrm{b}}$, A.S. Fokas ${ }^{\mathrm{b}, \mathrm{c}}$ \\ ${ }^{a}$ Sonny Astani Dept. of Civil and Environmental Engineering, University of Southern \\ California, Los Angeles, USA \\ ${ }^{b}$ Department of Applied Mathematics and Theoretical Physics, University of Cambridge, \\ Wilberforce Road, Cambridge, CB3 OWA, UK \\ ${ }^{c}$ Viterbi School of Engineering, University of Southern California, Los Angeles, CA 90089, \\ $U S A$
}

\begin{abstract}
This paper employs the unified transform, also known as the Fokas method, to solve the advection-dispersion equation on the half-line. This method combines complex analysis with numerics. Compared to classical approaches used to solve linear partial differential equations (PDEs), the unified transform avoids the solution of ordinary differential equations and more importantly constructs an integral representation of the solution in the complex plane which is uniformly convergent at the boundaries. As a consequence, such solutions are well suited for numerical computations. Indeed, the numerical evaluation of the solution requires only the computation of a single contour integral involving an integrand which decays exponentially fast for large values of the integration variable. A novel contribution of this paper with respect to the solution of linear evolution PDEs in general and the implementation of the unified transform in particular is the following: using the advection-dispersion equation as a generic example, it is shown that if the transforms of the given data can be computed analytically, then the unified transform yields a fast and accurate method that converges exponentially with the number of evaluations $N$ yet only has complexity $\mathcal{O}(N)$. Furthermore, if the transforms are computed numerically using $M$ evaluations,
\end{abstract}

\footnotetext{
${ }^{*}$ Corresponding author

Email addresses: fbarros@usc.edu (F.P.J. de Barros), m.colbrook@damtp.cam.ac.uk (M.J. Colbrook)
} 
the unified transform gives rise to a method with complexity $\mathcal{O}(N M)$. Results are successfully compared to other existing solutions.

Keywords: Unified Transform, Advection-Dispersion Equation, Fokas Method, Analytical Solution, Environmental Flows

\section{Introduction}

Several problems in physics and engineering are modelled by means of the advection-dispersion equation (ADE). Starting from the pioneering work of Taylor [1, the ADE has been used to predict transport of passive or reactive

5 scalars in natural (e.g. atmospheric, subsurface and surface flows) as well as in confined (e.g., channels and tubes) environments. Given its importance in many fields in science and engineering, it is not surprising that there exists a plethora of analytical solutions for the ADE under different physical configurations. In this work, we focus on the use of the ADE in environmental flows such as those occurring in natural porous formations (e.g., groundwater) and surface water bodies (e.g., rivers and channels).

There have been significant efforts in developing analytical solutions for the ADE in the environmental engineering and hydrological communities. For example, Ogata and Banks [2], van Genuchten and Alves [3], Kreft and Zuber [4],

15 Lee [5] and Guerrero et al. [6, 7], provide a compendium of analytical solutions for ADE that are applicable to fully saturated porous media. Other works [8, 9, 10] have derived analytical and semi-analytical solutions in order to study solute transport in a fractured porous medium. With the goal of predicting transport in geological formations displaying heterogeneities, analytical solutions have also been applied to estimate the ensemble mean concentration (see [11] and [12] for details). Similarly, analytical solutions exist for contaminant mixing in rivers [13, 14, 15, 16] and for bed-load sediment transport [17].

All of the above works rely on classical methods for solving linear partial differential equations, namely separation of variables and specific integral transforms 25 [18, 19, 20]. However, as explained in [21, the traditional transforms have 
several disadvantages which will be discussed below using the diffusion equation as an illustrative example.

Consider the diffusion equation on the half-line, with Dirichlet boundary conditions:

$$
\begin{aligned}
\frac{\partial \phi(x, t)}{\partial t} & =\frac{\partial^{2} \phi(x, t)}{\partial x^{2}}, 0<x<\infty \\
\phi(x, 0) & =\phi_{o}(x), 0<x<\infty ; \phi(0, t)=g_{o}(t), \quad t>0,
\end{aligned}
$$

30 $g_{o}(t)$ are given functions and $\phi_{o}(x) \rightarrow 0$ as $x \rightarrow \infty$. Using the sine transform, one finds the solution representation

$$
\phi(x, t)=\frac{2}{\pi} \int_{0}^{\infty} \sin (\lambda x) e^{-\lambda^{2} t}\left[\int_{0}^{\infty} \sin (\lambda \xi) \phi_{o}(\xi) d \xi-\int_{0}^{t} e^{\lambda^{2} \tau} g_{o}(\tau) d \tau\right] d \lambda
$$

1. The traditional approaches construct solutions which are not uniformly convergent at the boundaries unless the boundary conditions are homogeneous. In the particular case of equation (2), one cannot take the limit $x \rightarrow 0$ inside the integral otherwise the solution vanishes $\operatorname{since} \sin (\lambda x)$ vanishes for $x=0$.

2. Because of 1 , it is not straightforward to verify that the function $\phi(x, t)$ defined by the right hand side of (2) satisfies the boundary condition $\phi(0, t)=g_{o}(t)$.

3. Because of 1 , the solution obtained from traditional methods is not directly suitable for numerical computations.

4. The traditional approaches require separability of the partial differential equation, domain and boundary conditions.

455 . It is in general difficult to derive the proper transform.

6. Traditional transforms exist only for few boundary value problems. For example, there does not exist an $x$-transform for the equation $\frac{\partial \phi(x, t)}{\partial t}+$ $\frac{\partial^{3} \phi(x, t)}{\partial x^{3}}=0$, see $[22,23]$. 
The unified transform [21], also known as the Fokas method, has been used

so to obtain solutions for the diffusion equation [24, 25, 26], the reactive-diffusion equation [27, and recently has been applied to a physical problem with non-separable boundary conditions [28, as well as to interface problems [23, 22]. However the Fokas method has not been implemented to advective-dispersive (or convective-diffusive) problems.

55 In this work, we focus on solving the ADE via the unified transform. This work is structured as follows. Section 2 provides the formulation of the relevant physical-mathematical problem. The methodology is presented in Section 3 and specific semi-analytical solutions are obtained in Section 4. Computational results are illustrated and discussed in Section 5 with particular emphasis on the

6o speed and order of convergence of the method. Finally, a summary is provided in Section 6 .

\section{Problem Formulation}

Consider a one-dimensional semi-infinite medium with space and time variables $x$ and $t$. The concentration of an inert solute is assumed to be governed by the

$65 \mathrm{ADE}$

$$
\frac{\partial C(x, t)}{\partial t}+u \frac{\partial C(x, t)}{\partial x}=D \frac{\partial^{2} C(x, t)}{\partial x^{2}}, \quad 0<x<\infty,
$$

subject to the following boundary and initial conditions:

$$
\begin{aligned}
& C(0, t)=g_{o}(t), \\
& C(x, t) \rightarrow 0, \quad \text { as } x \rightarrow \infty, \quad t \geq 0, \\
& C(x, 0)=C_{o}(x),
\end{aligned}
$$

where $C\left[\mathrm{M} / \mathrm{L}^{3}\right]$ denotes the concentration of an inert solute, $u[\mathrm{~L} / \mathrm{T}]$ represents the constant velocity and $D\left[\mathrm{~L}^{2} / \mathrm{T}\right]$ is the dispersion coefficient. The problem defined by equations (3)-(6) can represent solute transport in a river for which 
a time variable concentration $g_{o}(t)$ is injected at the origin at time zero. It can also represent transport of a non-reactive substance through a fully saturated soil column.

\section{Methodology}

Our starting point is the definition of the Fourier transform pair,

$$
\begin{array}{ll}
\widehat{C}(\lambda, t)=\int_{0}^{\infty} e^{-\imath \lambda x} C(x, t) d x, & \operatorname{Im}\{\lambda\} \leq 0, \\
C(x, t)=\frac{1}{2 \pi} \int_{-\infty}^{\infty} e^{\imath \lambda x} \widehat{C}(\lambda, t) d \lambda & 0<x<\infty,
\end{array}
$$

with $\imath$ denoting the imaginary unit and $\lambda$ is a complex variable.

In order to obtain a solution to equation (3), we will proceed with the following methodological procedure proposed by Fokas [29, 21].

1. Employ the Fourier transform to obtain the global relation (GR). This equation provides an expression that couples the function $C(x, t)$ with its

80 derivatives on the boundary of the domain.

2. Obtain an integral representation of the solution $C(x, t)$ in the complex $\lambda$-plane.

3. By using the symmetry properties of the GR, eliminate from the integral representation of $C(x, t)$ (obtained in Step 2) the transforms of the unknown boundary values.

Next, we employ the above steps to construct a solution for the ADE (3) subject to the initial and boundary conditions (4)-(6).

Employing equation (7) into (3) and integrating by parts we find the following GR:

$$
e^{\left(\lambda^{2} D+\imath \lambda u\right) t} \widehat{C}(\lambda, t)=\widehat{C}_{o}(\lambda)-\widetilde{g}_{1}(\lambda, t)-\imath \lambda \widetilde{g}_{o}(\lambda, t), \quad \operatorname{Im}\{\lambda\} \leq 0,(9)
$$

so where, 


$$
\begin{aligned}
& \widehat{C}_{o}(\lambda)=\int_{0}^{\infty} e^{-\imath \lambda x} C_{o}(x) d x, \quad \operatorname{Im}\{\lambda\} \leq 0 \\
& \widetilde{g}_{1}(\lambda, t)=\int_{0}^{t} e^{\left(\lambda^{2} D+\imath \lambda u\right) \tau}\left[\left.D\left(\frac{\partial C}{\partial x}\right)\right|_{x=0}-u g_{o}(\tau)\right] d \tau, \lambda \in \mathbb{C}, t>0( \\
& \widetilde{g}_{o}(\lambda, t)=D \int_{0}^{t} e^{\left(\lambda^{2} D+\imath \lambda u\right) \tau} g_{o}(\tau) d \tau, \quad \lambda \in \mathbb{C}, t>0
\end{aligned}
$$

The derivation of (9) follows precisely the same steps used in the usual transform methods; details can be found in Appendix A.

Solving the GR 99 for $\widehat{C}(\lambda, t)$ and employing the inverse Fourier transform (8), we find

$$
\begin{aligned}
C(x, t) & =\frac{1}{2 \pi} \int_{-\infty}^{\infty} e^{\imath \lambda x-\left(\lambda^{2} D+\imath \lambda u\right) t} \widehat{C}_{o}(\lambda) d \lambda \\
& -\frac{1}{2 \pi} \int_{-\infty}^{\infty} e^{\imath \lambda x-\left(\lambda^{2} D+\imath \lambda u\right) t}\left[\widetilde{g}_{1}(\lambda, t)+\imath \lambda \widetilde{g}_{o}(\lambda, t)\right] d \lambda .
\end{aligned}
$$

In summary, using the transform pair (7) and (8) and following the traditional transform methodology, we obtain an integral representation for the scalar quantity $C(x, t)$. However, the representation 13 involves the $t$-transform of the unknown boundary value $\partial C /\left.\partial x\right|_{x=0}$ defined by the function $\widetilde{g}_{1}(\lambda, t)$ in 11. In order to eliminate this transform, we will adopt the two novel steps described below.

First, we deform the contour of the second integral in the right-hand-side of equation 13 from the real axis to the upper half of the complex-plane. In this connection, we need to determine the domain in the $\lambda$-complex plane where $\exp \left[\imath \lambda x-\left(\lambda^{2} D+\imath \lambda u\right)(t-\tau)\right]$ is bounded (for $x \geq 0$ and $(t-\tau) \geq 0$ ). This 105 can be achieved by analyzing the exponentials exp $[\imath \lambda]$ and $\exp \left[-\left(\lambda^{2} D+\imath \lambda u\right)\right]$ : the exponential $\exp [\imath \lambda]$ is bounded for $\operatorname{Im}\{\lambda\} \geq 0$. We also note that $\lambda^{2} D+$ $\imath \lambda u=D\left(\lambda_{R}^{2}-\lambda_{I}^{2}+2 \imath \lambda_{R} \lambda_{I}\right)+\imath u\left(\lambda_{R}+\imath \lambda_{I}\right)$, where $\lambda=\lambda_{R}+\imath \lambda_{I}$. Thus, $\operatorname{Re}\left\{\lambda^{2} D+\imath \lambda u\right\}=D\left(\lambda_{R}^{2}-\lambda_{I}^{2}\right)-u \lambda_{I}$. Examining the curve

$$
D\left(\lambda_{R}^{2}-\lambda_{I}^{2}\right)-u \lambda_{I}=0
$$




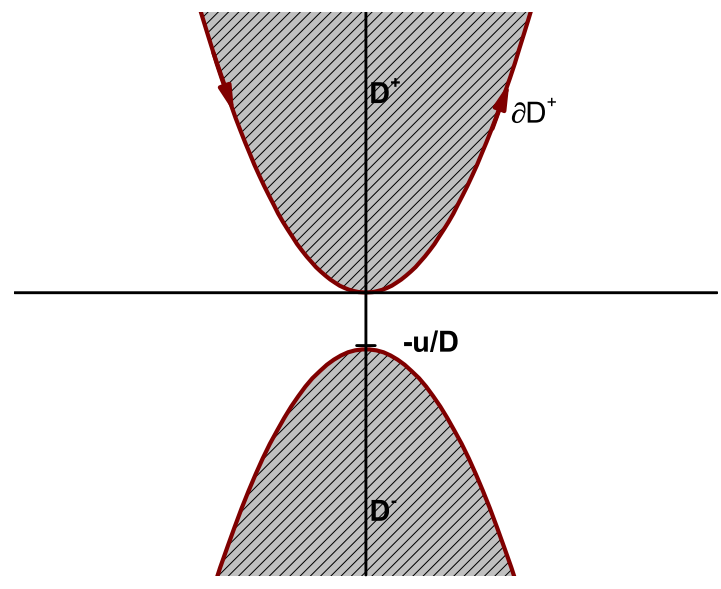

Figure 1: Boundaries $\partial D^{+}$and $\partial D^{-}$of the domains $D^{+}$and $D^{-}$which are defined by $D^{ \pm}=\left\{\lambda=\lambda_{R}+\imath \lambda_{I}, \lambda_{R} \in \mathbb{R}, \lambda_{I} \in \mathbb{R}, \operatorname{Re}\left\{\lambda^{2} D+\imath u \lambda\right\} \lessgtr 0\right\}$.

we note that if $\lambda_{I}=0$, then $\lambda_{R}=0$. Therefore, if $\lambda_{R}=0$ then $\lambda_{I}=0$ or ${ }_{110} \lambda_{I}=-u / D$. Also, we observe that $\lambda_{I}^{2}+(u / D) \lambda_{I} \geq 0$ or $\lambda_{I}\left(\lambda_{I}+u / D\right)=0$, hence, either $\lambda_{I} \geq 0$ or $\lambda_{I}<-u / D$. Thus, we can replace the contour in the second term of the right-hand-side of $(13)$ with the contour $\partial D^{+}$in Figure 1. where the curve $\partial D^{+}$is defined by

$$
D\left(\lambda_{R}^{2}-\lambda_{I}^{2}\right)-u \lambda_{I}=0, \quad \text { with } \lambda=\lambda_{R}+\imath \lambda_{I}
$$

Both functions $\widetilde{g}_{1}(\lambda, t)$ and $\widetilde{g}_{o}(\lambda, t)$ (see equations (11) and (12)) depend on $115 \lambda$ only through the combination $\lambda^{2} D+\imath \lambda u$. This fact motivates the search for a transformation $\lambda \rightarrow v$ such that the above combination is invariant:

$$
\lambda^{2} D+\imath \lambda u=v^{2} D+\imath v u
$$

or

$$
\left(\lambda^{2}-v^{2}\right) D+\imath u(\lambda-v)=0 .
$$


Hence,

$$
(\lambda-v)[(\lambda+v) D+\imath u]=0 .
$$

Thus, $v=\lambda$ or $v=-\lambda-\imath u / D$. Replacing $\lambda$ by $-\lambda-\imath u / D$ in the global relation 120 (9) we obtain:

$$
e^{\left(\lambda^{2} D+\imath \lambda u\right) t} \widehat{C}\left(-\lambda-\imath \frac{u}{D}, t\right)=\widehat{C}_{o}\left(-\lambda-\imath \frac{u}{D}\right)-\widetilde{g}_{1}(\lambda, t)+\left(\imath \lambda-\frac{u}{D}\right) \widetilde{g}_{o}(\lambda, t) .
$$

Solving (19) for $\widetilde{g}_{1}$, substituting the resulting equation in 13 with the integration in the second term replaced by the integration along the contour $\partial D^{+}$, we obtain the equation

$$
\begin{aligned}
C(x, t)= & \frac{1}{2 \pi} \int_{-\infty}^{\infty} e^{\imath \lambda x-\left(\lambda^{2} D+\imath \lambda u\right) t} \widehat{C}_{o}(\lambda) d \lambda \\
- & \frac{1}{2 \pi} \int_{\partial D^{+}} e^{\imath \lambda x-\left(\lambda^{2} D+\imath \lambda u\right) t} \times \\
& \left\{\widehat{C}_{o}\left(-\lambda-\imath \frac{u}{D}\right)-\widehat{C}\left(-\lambda-\imath \frac{u}{D}, t\right) e^{\left(\lambda^{2} D+\imath \lambda u\right) t}+\left(2 \imath \lambda-\frac{u}{D}\right) \widetilde{g}_{o}(\lambda, t)\right\} d \lambda .
\end{aligned}
$$

It is important to note that the unknown term $\widehat{C}(-\lambda-\imath u / D, t)$ does not contribute to the final expression, since both this term and $\exp (\imath \lambda x)$ are bounded and analytic in $D^{+}$. Furthermore, $\widehat{C}(-\lambda-\imath u / D, t)$ decays as $\lambda \rightarrow \infty$. Thus, according to Cauchy's theorem, the integral

$$
\int_{\partial D^{+}} e^{\imath \lambda x} \widehat{C}\left(-\lambda-\imath \frac{u}{D}, t\right) d \lambda
$$

vanishes. Therefore, the general solution of the ADE (3) subject to the conditions

125 (4)-(6) is given by

$$
\begin{aligned}
C(x, t) & =\frac{1}{2 \pi} \int_{-\infty}^{\infty} e^{\imath \lambda x-\left(\lambda^{2} D+\imath \lambda u\right) t} \widehat{C}_{o}(\lambda) d \lambda \\
& -\frac{1}{2 \pi} \int_{\partial D^{+}} e^{\imath \lambda x-\left(\lambda^{2} D+\imath \lambda u\right) t}\left[\widehat{C}_{o}\left(-\lambda-\imath \frac{u}{D}\right)+\left(2 \imath \lambda-\frac{u}{D}\right) \widetilde{g}_{o}(\lambda, t)\right] d \lambda,
\end{aligned}
$$


where the contour $\partial D^{+}$is depicted in Figure 1 and defined by $(15)$.

\section{Specific Solutions}

We illustrate several features of the analytical expression derived in Section 3. Using equation (21) as a starting point, we develop solutions for two specific cases of increasing level of complexity.

\subsection{Test Case 1 - Continuously Injected Solute at Constant Concentration}

For our first demonstration, we set in equations (4) and (6) $g_{o}(t)=C_{i}$ and $C_{o}(x)=0$. This situation represents, for example, advective-dispersive transport of a solute through a homogeneous, fully saturated, porous medium where an inactive tracer is continuously injected at the origin with inlet concentration $C_{i}$, i.e., these conditions are met in laboratory scale tracer experiments in soil columns. Under the above conditions, equation (21) becomes

$$
C(x, t)=-\frac{1}{2 \pi} \int_{\partial D^{+}} e^{\imath \lambda x-\left(\lambda^{2} D+\imath \lambda u\right) t}\left(2 \imath \lambda-\frac{u}{D}\right) \widetilde{g}_{o}(\lambda, t) d \lambda,
$$

with

$$
\begin{aligned}
\widetilde{g}_{o}(\lambda, t) & =C_{i} D \int_{0}^{t} e^{\left(\lambda^{2} D+\imath \lambda u\right) \tau} d \tau \\
& =C_{i} D\left[\frac{e^{\left(\imath u \lambda+D \lambda^{2}\right) t}-1}{\lambda(\imath u+D \lambda)}\right] .
\end{aligned}
$$

Using (22) and (23), the equation satisfied by the scalar $C(x, t)$ becomes

$$
C(x, t)=-\frac{C_{i} D}{2 \pi} \int_{\partial D^{+}} e^{\imath \lambda x-\left(\lambda^{2} D+\imath \lambda u\right) t} \frac{\left(2 \imath \lambda-\frac{u}{D}\right)}{\lambda(\imath u+D \lambda)}\left[e^{\left(\lambda^{2} D+\imath \lambda u\right) t}-1\right] d \lambda,
$$

140 which can be further simplified to 


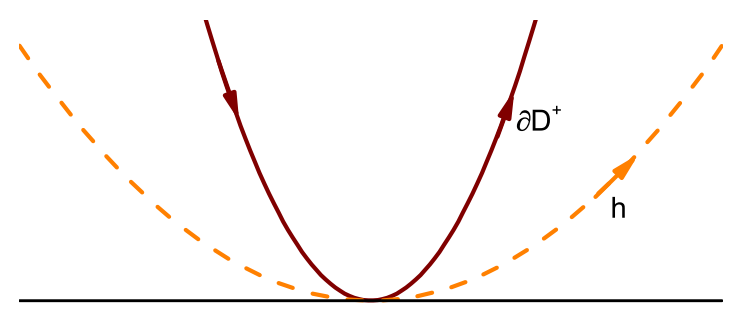

Figure 2: Schematic of the contour $h$.

$$
C(x, t)=-\frac{\imath C_{i}}{2 \pi} \int_{\partial D^{+}}\left(\frac{2 D \lambda+\imath u}{D \lambda+\imath u}\right) e^{\imath \lambda x} \frac{\left[1-e^{-\left(\lambda^{2} D+\imath \lambda u\right) t}\right]}{\lambda} d \lambda,
$$

where the integration over $\partial D^{+}$will be carried out numerically. In this connection, it is convenient (using Cauchy's theorem) to replace $\partial D^{+}$by the contour $h$ depicted below (see Figure 21). This contour has the advantage that now not only $\exp [\imath \lambda x]$ decays for large $\lambda$, but also $\exp \left[-\left(\lambda^{2} D+\imath \lambda u\right) t\right]$ which oscillates on the contour $\partial D^{+}$, decays on the contour $h$.

In summary, equation 25 is the solution for the initial boundary value problem subject to $g_{o}(t)=C_{i}$ and $C_{o}(x)=0$ and will be used to compute the concentration field. Additional details regarding the numerical integration is provided in Section 5. It is shown in Appendix B that equation 25 can be 150 simplified further.

The problem under consideration, i.e. $g_{o}(t)=C_{i}$ and $C(x, 0)=0$, has been tackled in the past by Ogata and Banks [2. In order to obtain a solution for this problem, Ogata and Banks [2] mapped the ADE into a diffusion equation and then made use of Duhamel's theorem [18] to derive the following approximate analytical expression:

$$
C(x, t) \approx \frac{C_{i}}{2}\left\{\operatorname{Erfc}\left[\frac{x-u t}{2 \sqrt{D t}}\right]+e^{\frac{u x}{D}} \operatorname{Erfc}\left[\frac{x+u t}{2 \sqrt{D t}}\right]\right\}
$$

We will utilize equation (26), which represents an approximate solution for the concentration field, to benchmark the results obtained through the unified 
transform (computed by using equation (25)).

\subsection{Test Case 2 - Time Variable Boundary Condition and Spatially Distributed}

\section{Initial Condition}

Next, we derive the solution for the following case: $g_{o}(t)=\mathcal{A}_{i}[\cos (\omega t)+1]$ and $C_{o}(x)=\exp [-x]$ (see equations (4) and (6)) where $\mathcal{A}_{i}$ and $\omega$ are amplitude and angular frequency respectively. Under this condition, both $\widehat{C}_{o}(\lambda)$ and $g_{o}(t)$ (see equation (21)), can be computed analytically. Explicit expressions for $\widehat{C}_{o}(\lambda), \widehat{C}_{o}(-\lambda-\imath u / D)$ and $g_{o}(t)$ are provided below:

$$
\begin{aligned}
\widehat{C}_{o}(\lambda)= & -\frac{\imath}{\lambda-\imath}, \\
\widehat{C}_{o}\left(-\lambda-\imath \frac{u}{D}\right)= & \frac{\imath D}{\imath u+D(\imath+\lambda)} \\
\widetilde{g}_{o}(\lambda, t)= & D \mathcal{A}_{i}\left\{\left[\frac{e^{\lambda t(\imath u+D \lambda)}-1}{\lambda(\imath u+D \lambda)}\right]+\right. \\
& \left.\left\{\frac{e^{\lambda t(\imath u+D \lambda)}[\lambda(\imath u+D \lambda) \cos (\omega t)+\omega \sin (\omega t)]-\lambda(\imath u+D \lambda)}{\lambda^{2}(\imath u+D \lambda)^{2}+\omega^{2}}\right\}\right\} .
\end{aligned}
$$

Substituting these into (21) leads to

$$
C(x, t)=\frac{1}{2 \pi}\left[\mathcal{P}_{1}(x, t)-\mathcal{P}_{2}(x, t)\right],
$$

where

$$
\begin{aligned}
\mathcal{P}_{1}(x, t)= & -\int_{-\infty}^{\infty} e^{\imath \lambda x-\left(\lambda^{2} D+\imath \lambda u\right) t}\left(\frac{\imath}{\lambda-\imath}\right) d \lambda \\
\mathcal{P}_{2}(x, t)= & \left\{\mathcal{A}_{i} \int_{\partial D^{+}} e^{\imath \lambda x-\left(\lambda^{2} D+\imath \lambda u\right) t}(2 \imath \lambda D-u)\left[\frac{e^{\lambda t(\imath u+D \lambda)}-1}{\lambda(\imath u+D \lambda)}\right] d \lambda\right. \\
+ & \int_{\partial D^{+}} e^{\imath \lambda x-\left(\lambda^{2} D+\imath \lambda u\right) t}\left[\frac{D \imath}{\imath u+D(\imath+\lambda)}+\mathcal{A}_{i}(2 \imath \lambda D-u) \times\right. \\
& \left.\left.\frac{e^{\lambda t(\imath u+D \lambda)}[\lambda(\imath u+D \lambda) \cos (\omega t)+\omega \sin (\omega t)]-\lambda(\imath u+D \lambda)}{\lambda^{2}(\imath u+D \lambda)^{2}+\omega^{2}}\right] d \lambda\right\} .
\end{aligned}
$$


In summary, equation $(30)$ provides the solution of the initial boundary value problem with $g_{o}(t)=\mathcal{A}_{i}[\cos (\omega t)+1]$ and $C_{o}(x)=\exp [-x]$. Similar to Test

170 Case 1 , the contours in (31) and 32 can be replaced by the contour $h$ depicted in Figure 2 which is used in the numerical evaluations and defined in the next section.

\section{Computational Results}

For the upcoming computational illustrations, we plot the results for Test

175 Cases 1 and 2 in dimensionless form. The following dimensionless quantities were adopted for the concentration $C$, distance $x$ and time $t$ respectively:

$$
\chi=\frac{C}{C_{i}} \quad ; \quad \xi=\frac{x u}{D} ; \quad \eta=\frac{u^{2} t}{D}
$$

with $C_{i}$ denoting a characteristic concentration value at the inlet boundary.

The analytic solution of the solute concentration (after a suitable parametrization) can be represented in terms of integrals of the following two forms:

$$
L_{1}(f, \alpha)=\int_{0}^{\infty} f(x) e^{-\alpha x} d x, \quad L_{2}(f, \alpha)=\int_{-\infty}^{\infty} f(x) e^{-\alpha x^{2}} d x,
$$

where the functions $f$ are well behaved (smooth and bounded). In the case that $\alpha$ is not small, we shall evaluate these integrals using Gauss-Laguerre quadrature 180 for $L_{1}$ and Gauss-Hermite quadrature for $L_{2}$. For the functions considered in this paper these quadrature rules will converge exponentially (we refer the reader to [30, 31, 32] for the theory of these quadrature rules) with the number of evaluations, which we denote throughout by $N$. These quadrature rules (the weights and nodes) can also be computed extremely fast in $\mathcal{O}(N)$ operations 185 33, 34, 35. Similarly, if $\alpha$ is small (as in the case of 25 for small $x$ ) we shall truncate the domain of integration (typically to $|\lambda|<100$ ) and apply standard Gaussian quadrature which will also converge exponentially and have $\mathcal{O}(N)$ cost. In all cases (assuming the functions $\widehat{C}_{o}$ and $\widetilde{g}_{o}$ are known), we recover a quadrature method which can be implemented in $\mathcal{O}(N)$ operations per point 
$(x, t)$ which will also converge pointwise exponentially in $N$. Note that this is a key advantage of the unified transform over classical methods as mentioned in the introduction. We will also consider the case where $\widehat{C}_{o}$ and $\widetilde{g}_{o}$ themselves must be computed in Section 5.3 .

All computational results were obtained using MATLAB on a standard desktop computer and to compare we have computed accurate reference solutions using adaptive Gauss-Kronrod quadrature to evaluate the integrals in the unified transform (with tolerance set to machine precision). For the numerical evaluations of the concentration field through equation 21, the numerical computation of the integrals in 25) and 30 is achieved by deforming $\partial D^{+}$to a contour $h$ between the real line and $\partial D^{+}$(see Figure 2). A convenient choice for $h$ is the union of the two line segments

$$
\begin{aligned}
& \{\ell[\cos (\pi-\theta)+\imath \sin (\pi-\theta)]: \ell \geq 0\}, \\
& \{\ell[\cos (\theta)+\imath \sin (\theta)]: \ell \geq 0\},
\end{aligned}
$$

where $\theta$ is the angle between the line segment and the real line. For our

195 illustrations, we set $\theta=\pi / 6$.

\subsection{Results for Test Case 1}

As a first example, we consider the computation of the solution for $\eta=16$. Figure 3 shows the computed solution using equation B11 with $N=50$ quadrature points, denoted as Approach 1 in the plot, and the approximate analytical solution 26) obtained through traditional methods. For completeness, we also include a comparison with a numerical solution based on the Crank-Nicolson and backward Euler finite-difference methods with $\Delta t=\Delta x=h=0.5$. In addition, Figure 3 compares with a solution, also based via the unified transform, obtained by mapping the ADE into a diffusion equation (denoted as Approach

2052 in the plot). The unified transform-based solution for the diffusion equation is well documented in the literature [24, 21] and for the sake of brevity it is not reproduced here. All of the methods agree well. 


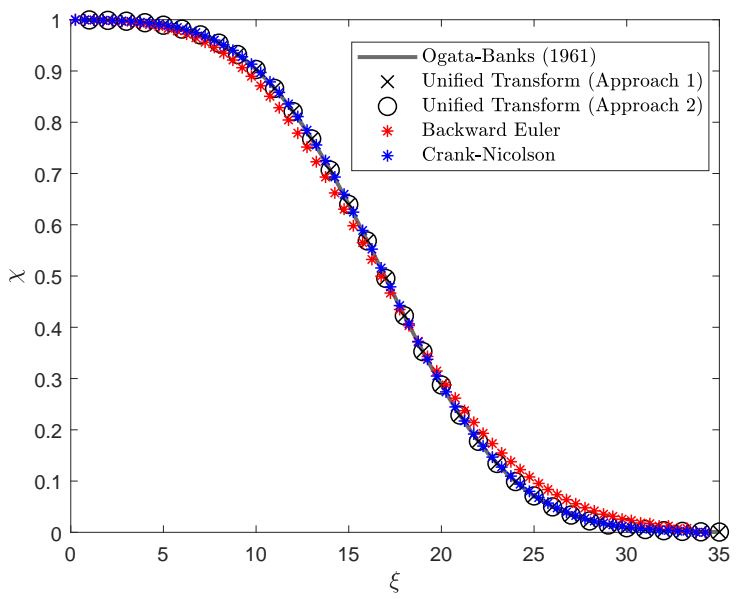

Figure 3: Dimensionless concentration $\chi(\xi, \eta)$ versus distance $\xi$ at $\eta=16$. Comparison between the proposed analytical solution with other analytical and numerical solutions. Approach 1 refers to the solution given by B11 (which can also be obtained via 25) for $N=50$. Approach 2 refers to the solution obtained through the unified transform method by mapping the ADE into a diffusion equation. We have also shown the solutions obtained using Crank-Nicolson and backward Euler finite-difference methods with $h=0.5$ (note the increased accuracy in using Crank-Nicolson). 

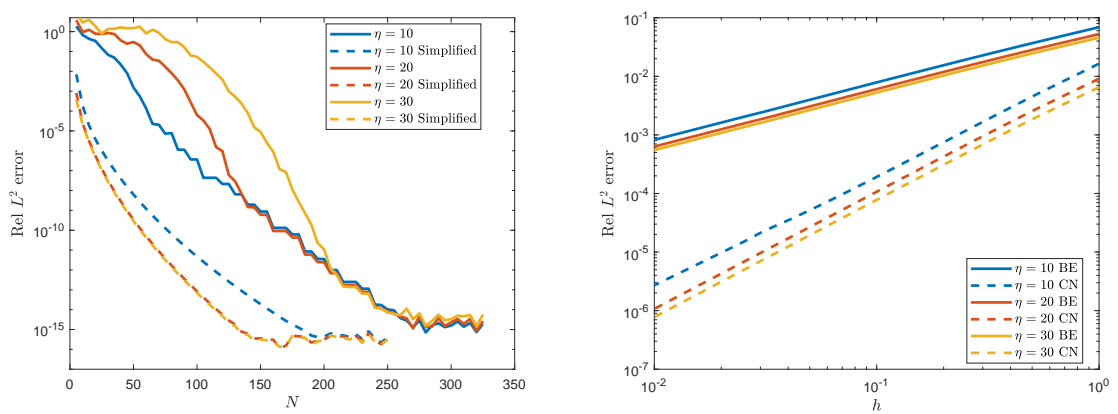

Figure 4: Left: Exponential convergence of the unified transform as measured via a relative $L^{2}$ norm. We have shown convergence using both 25 and the simplified version B11. Right: Algebraic convergence of Crank-Nicolson (CN, order 2) and backward Euler (BE, order 1) finite-difference methods.

Figure 4 shows the convergence of the method for different $\eta$ as we increase $N$, where we have approximated the relative error measured in $L^{2}$ norm (i.e. normalised by the $L^{2}$ error of the true solution). As expected, we see exponential convergence in $N$, with the simplified version requiring smaller $N$ (see Figure 4 left). Interestingly, whilst we need larger $N$ for larger times to achieve modest errors, there seems to be a cut-off beyond which the errors depend similarly with respect to $N$ independent of $\eta$. To verify our solution, we have also shown methods (see Figure 4 right). For the finite-difference methods we note that one needs to solve a triangular $M \times M$ system at each step where $M \approx \mathcal{O}\left(h^{-1}\right)$. This can be done in $\mathcal{O}(M)$ operations as opposed to $\mathcal{O}\left(M^{3}\right)$ for a full system but still gives $\mathcal{O}\left(h^{-1} \Delta t^{-1}\right)$ operations to compute the solution at any fixed 220 time $t$ and the methods only converge algebraically. This shows a significant advantage of the unified transform: as well as obtaining the solution at any time without time-stepping, the integral representation lends itself to extremely efficient numerical integration.

Next, we consider the computation of the solution over a range of values of $\xi$ and $\eta$. Figure 5 (top) shows the concentration profile computed using the unified transform. As time increases, the front of the solute cloud advances through the 
flow domain and becomes more diffused with time. We have also shown the error of the method with $N=250$ using both 25 and B11 (see Figure 5 bottom left and bottom right respectively). The method is very fast, taking approximately 230 $0.29 \mathrm{~s}$ using 25 and $0.08 \mathrm{~s}$ using (B11) to compute the profile over 4800 points. Using 25 is slower due to the need to compute two integrals. In comparison, the reference solution was computed in 19s (with adaptive quadrature). Both sets of errors approach machine precision over a large portion of the domain. Using B11 appears to struggle near the singularity at $(\xi, \eta)=(0,0)$ (this is to be expected from the blow up of the integrand) and (25) appears to struggle more for small $\xi$, particularly at larger $\eta$ due the need to evaluate a slowly (but still exponentially) decaying oscillatory integrand. We found increasing $N$ allows more accurate solutions to be computed near the boundary. We also remark that the concentration profile computed through the unified transform

240 almost perfectly matches the solution computed through different approaches such as the approximate solution given by 26] 2]. The approximation 26 , was found to agree to about 12 decimal places in the domain interior.

Finally, Figure 6 shows the value of $N$ needed in order to ensure five digits of accuracy, as a function of $\xi$. This shows that accurate solutions can be computed 245 extremely efficiently, especially with the integral form B11.

\subsection{Results for Test Case 2}

Now we present the results obtained from equation f30 for an oscillatory boundary condition and an exponentially distributed initial condition. For this analysis, we set $\omega=1$ and $\mathcal{A}_{i}=C_{i} / 2$ in equations 27 and 29p. Figure 7 250 shows the concentration as a function of distance for different times $(\eta=5$, 10, 20 and 30). In addition, Figure 7 compares the results computed from the unified transform 30 with the numerical solution of the ADE subject to the conditions described in Section 4.2. The numerical solution was computed using MATLAB's built-in function pdepe which runs a variable time-stepping

255 method, second order in the spatial resolution [36. As shown in Figure 7, both methods agree well. 

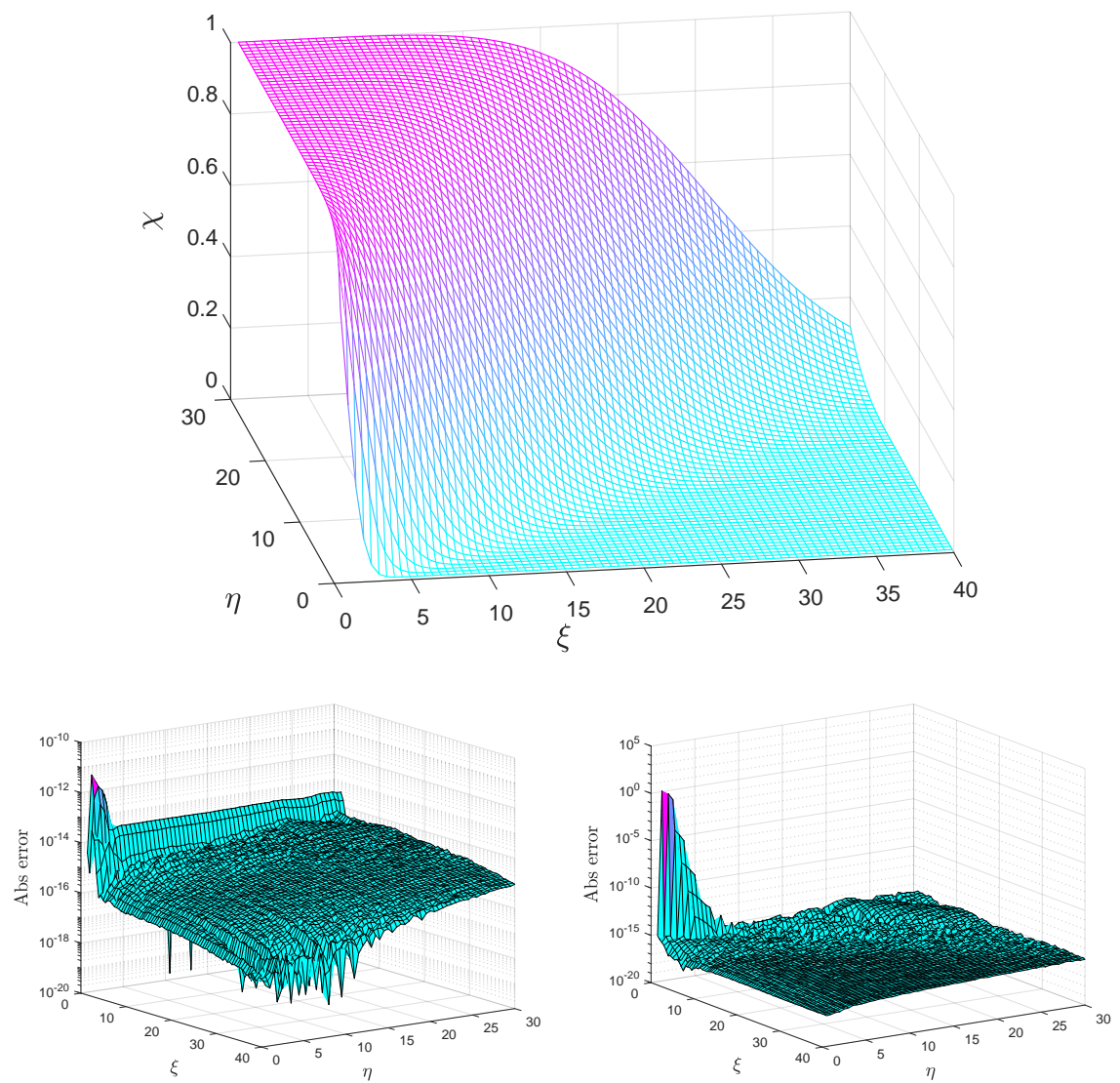

Figure 5: Top: Concentration profile. Bottom left: Absolute error when using 25]. Bottom right: Absolute error when using B11. 

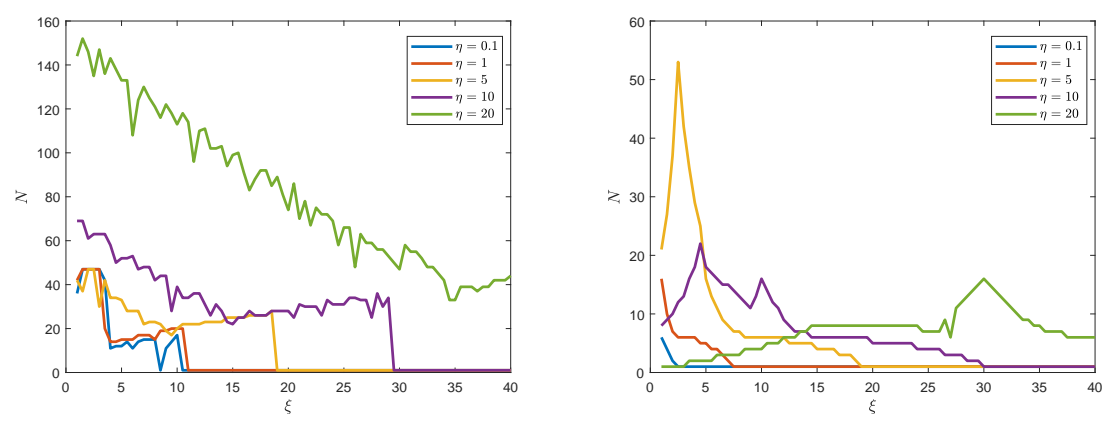

Figure 6: Left: Value of $N$ needed for five digits of accuracy when using 25. Right: Value of $N$ needed for five digits of accuracy when using B11.

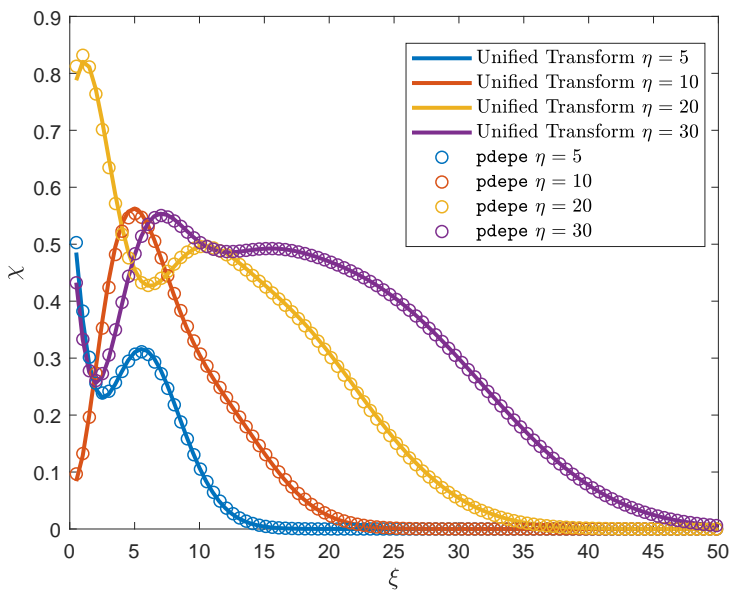

Figure 7: Dimensionless concentration $\chi(\xi, \eta)$ versus dimensionless distance $\xi$ for $\eta=5,10$, 20 and 30. Comparison between the proposed analytical solution with the fully numerical solution (computed using $h=0.5$ ). 

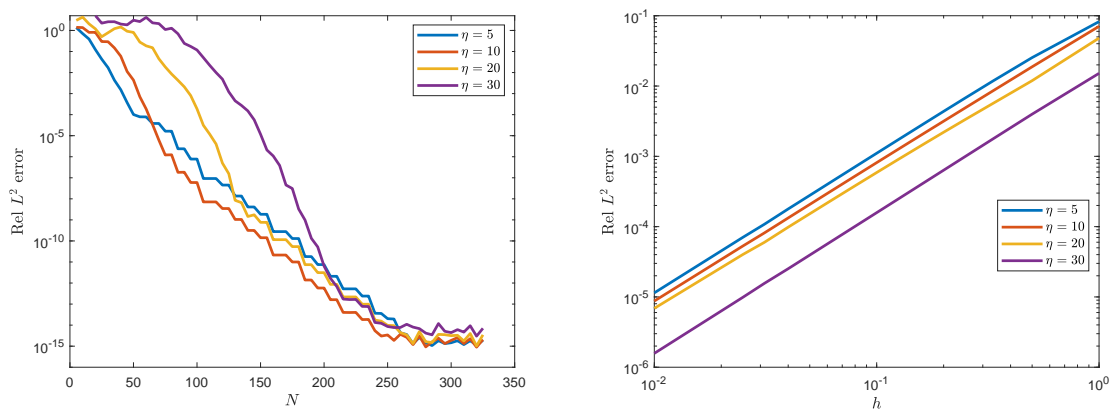

Figure 8: Left: Exponential convergence of the unified transform as measured via a relative $L^{2}$ norm for Case 2. Right: Algebraic convergence of MATLAB's pdepe (order 2).
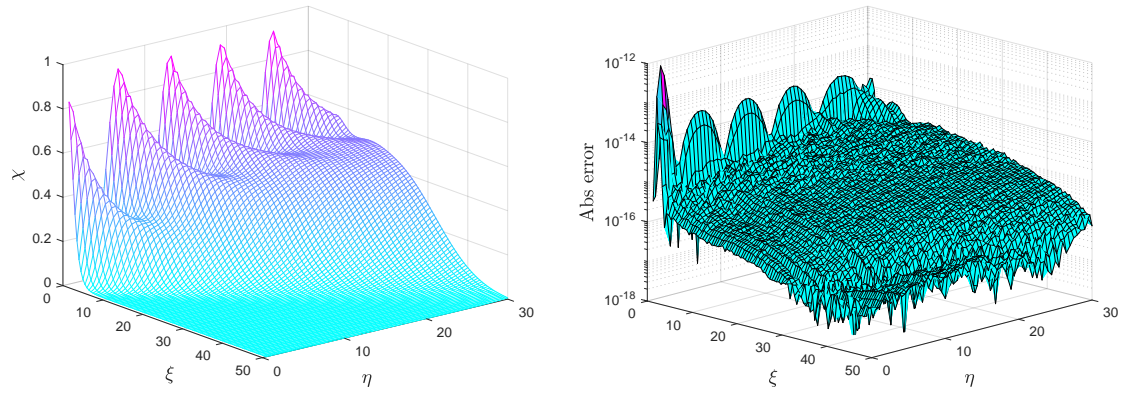

Figure 9: Left: Concentration profile. Right: Absolute error for $N=250$.

Figure 8 shows the convergence of the unified transform as we increase $N$. In the results depicted in Figure 8(left), we see exponential convergence as before. For completeness, we have also shown the convergence of pdepe as $h \downarrow 0$ (Figure 2608 right). As expected, the convergence of pdepe is algebraic with error of order $\mathcal{O}\left(h^{2}\right)$, however this still serves to verify the computation of the solution using the unified transform. Finally, we have shown the solution over a range of values of $\xi$ and $\eta$, as well as the error of the unified transform for $N=250$ in Figure 9 As before, we see errors close to machine precision over the domain, with larger errors nearer the boundary $\xi=0$. Again the method was very fast, taking $0.85 \mathrm{~s}$ for evaluation at 6000 points. 


\subsection{An Example where the Integral Transforms Cannot be Computed Analytically}

As a final example, we will consider the case where the functions $\widehat{C}_{o}$ and $\widetilde{g}_{o}$ themselves are computed numerically. Note that we only need to compute these at $\mathcal{O}(N)$ points. We will use Gaussian quadrature with $M_{1}$ nodes to compute $\widetilde{g}_{o}$. Suitable quadrature rules for the computation of $\widehat{C}_{o}$ depend on the decay properties of $C_{o}(x)$ as $x \rightarrow \infty$. We will consider the case of

$$
C_{o}(x)=\cos (x) \exp (-x)
$$

Note that $\widehat{C}_{o}$ can be computed analytically but instead we shall use Gaussian-Laguerre quadrature with $M_{2}$ nodes. Altogether, this leads to a method with computational $\operatorname{cost} \mathcal{O}\left(N\left(M_{1}+M_{2}\right)\right)$. In general, the error of such a method will depend on the error of the computation of the integrals (a full discussion of which is beyond the scope of this paper, but there is a large literature on the convergence methods of different quadrature rules). For this example we will take

$$
g_{o}(t)=J_{0}(t),
$$

where $J_{0}$ denotes the Bessel function of the first kind of order 0 . For these choices of functions, the error in the computation of the spectral functions decreases with an maximum error bounded by $\approx 2 \times 10^{-14}$. We verified this against the solution using pdepe where the solutions agreed to about five decimal places. However, the unified transform is able to gain much more accurate solutions. Finally, to examine how the error depends on varying $M_{1}$ and $M_{2}$, Table 1 shows 280 the average absolute error (over the same computational grid) with $N=500$ and various $M_{1}$ and $M_{2}$. We see that large $M_{1}$ is more critical to computing very accurate solutions as expected due to the exponential decay of $C_{o}$. 

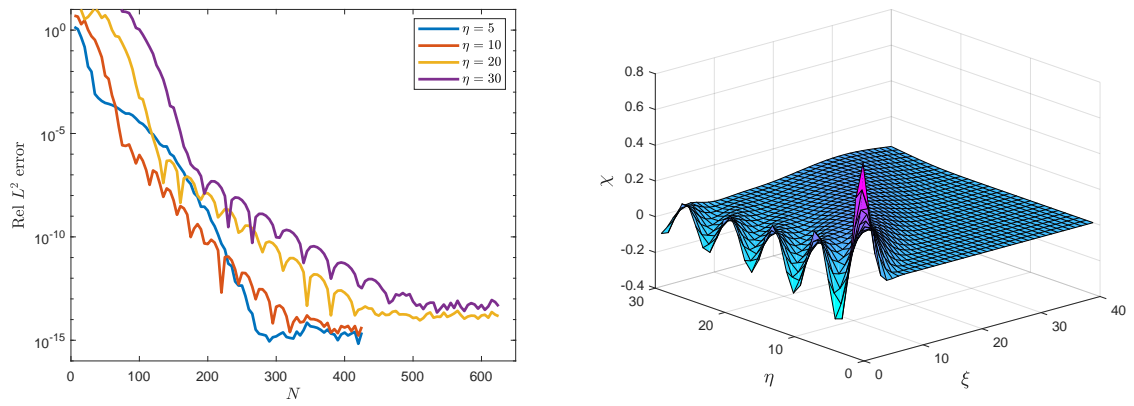

Figure 10: Left: Exponential convergence of the unified transform with increasing $N$ for the case of using quadrature to evaluate the spectral functions. Right: Computed solution over various $\xi$ and $\eta$. The maximum absolute error was bounded by $\approx 2 \times 10^{-14}$.

\begin{tabular}{|c|c|c|c|c|c|}
\hline$M_{2} M_{1}$ & 100 & 200 & 300 & 400 & 500 \\
\hline 40 & $3.49 \times 10^{-8}$ & $1.49 \times 10^{-10}$ & $8.02 \times 10^{-11}$ & $8.00 \times 10^{-11}$ & $8.00 \times 10^{-11}$ \\
\hline 80 & $3.48 \times 10^{-8}$ & $6.92 \times 10^{-11}$ & $2.53 \times 10^{-13}$ & $9.36 \times 10^{-15}$ & $5.90 \times 10^{-15}$ \\
\hline 120 & $3.48 \times 10^{-8}$ & $6.91 \times 10^{-11}$ & $2.48 \times 10^{-13}$ & $3.80 \times 10^{-15}$ & $3.35 \times 10^{-16}$ \\
\hline 160 & $3.48 \times 10^{-8}$ & $6.91 \times 10^{-11}$ & $2.48 \times 10^{-13}$ & $3.77 \times 10^{-15}$ & $3.18 \times 10^{-16}$ \\
\hline
\end{tabular}

Table 1: Average error for various $M_{1}$ and $M_{2}$. 


\section{Summary}

We have implemented the unified transform to obtain the solution of the

285 (constant and time varying). The unified transform method contains the classical approaches as special cases. In particular, we highlight the following key advantages of this new method: (1) it allows one to construct a representation of the solution which is uniformly convergent at the boundaries; (2) it can be implemented efficient for large times, since it does not require discretization of the time domain. The solution obtained by the new method is in excellent agreement with the computational results obtained through numerical methods and other analytical solutions. In particular, it gives rise to a numerical method converging exponentially as the number of evaluations $N$ increases and only has $\mathcal{O}(N)$ complexity.

Although the solution was obtained for advective-dispersive problems relevant for environmental engineering and hydrological applications, it can be employed to study a series of other applications regarding heat and mass transfer in mechanical, chemical, petroleum and biomedical engineering [e.g., 37. The solution obtained through the unified transform is well-suited to compute the concentration field from laboratory tracer experiments in soil columns where, in general, the velocity and dispersion coefficient are homogeneous. It is important to note that it is straightforward to implement the unified transform to problems transform is its applicability to a wide range of boundary conditions including Neumann, Robin and non-local boundary conditions. It can also be applied to some problems where classical methods apparently fail 40, 41 and PDEs involving third order spatial derivatives (see point 6 in the introduction). Multidimensional applications and extensions to reactive transport will be presented in future work.

Finally, it should be emphasised that the unified transform is the only 
existing method which expresses the solution of linear evolution PDEs in terms of an integral involving a contour in the $\lambda$-complex plane. This opens the way for an efficient numerical evaluation of the solution. In this paper, for the first time, the advantage of the numerical implementation of the unified transform is elucidated.

\section{Appendix A}

Multiplying equation (3) with $e^{-\imath \lambda x}$ and integrating with respect to $x$, we obtain

$\frac{\partial}{\partial t} \int_{0}^{\infty} e^{-\imath \lambda x} C(x, t) d x+\int_{0}^{\infty} e^{-\imath \lambda x} u \frac{\partial C(x, t)}{\partial x} d x=\int_{0}^{\infty} e^{-\imath \lambda x} D \frac{\partial^{2} C(x, t)}{\partial x^{2}} d x$.

This equation can be further simplified to

$$
\frac{\partial \widehat{C}(\lambda, t)}{\partial t}+u \int_{0}^{\infty} e^{-\imath \lambda x} \frac{\partial C(x, t)}{\partial x} d x=D \int_{0}^{\infty} e^{-\imath \lambda x} \frac{\partial^{2} C(x, t)}{\partial x^{2}} d x .
$$

Integration by parts of the second term in the left-hand side of $(\mathrm{A} 2$ yields

$$
u \int_{0}^{\infty} e^{-\imath \lambda x} \frac{\partial C(x, t)}{\partial x} d x=u[-C(0, t)+\imath \lambda \widehat{C}(\lambda, t)] .
$$

For the right-hand side of $\mathrm{A} 2$, we obtain the following expression after integration by parts:

$$
D \int_{0}^{\infty} e^{-\imath \lambda x} \frac{\partial^{2} C(x, t)}{\partial x^{2}} d x=D\left[-\left.\frac{\partial C(x, t)}{\partial x}\right|_{x=0}+\imath \lambda \int_{0}^{\infty} e^{-\imath \lambda x} \frac{\partial C(x, t)}{\partial x} d x\right]
$$

325 Integrating by parts again, we obtain

$$
D \int_{0}^{\infty} e^{-\imath \lambda x} \frac{\partial^{2} C(x, t)}{\partial x^{2}} d x=D\left[-\left.\frac{\partial C(x, t)}{\partial x}\right|_{x=0}-\imath \lambda C(0, t)-\lambda^{2} \widehat{C}(\lambda, t)\right] .
$$


Substituting equations $\mathrm{A} 3 \mathrm{n}$ and $\mathrm{A} 5 \mathrm{n}$ into $\mathrm{A} 2 \mathrm{y}$ yields

$$
\begin{aligned}
\frac{\partial \widehat{C}(\lambda, t)}{\partial t}+\left(\lambda^{2} D+\imath u \lambda\right) \widehat{C}(\lambda, t)= & -\left[\left.D \frac{\partial C(x, t)}{\partial x}\right|_{x=0}-u C(0, t)\right](\mathrm{A} 6) \\
& -\imath D \lambda C(0, t) .
\end{aligned}
$$

The above expression can be re-written as

$$
\begin{aligned}
\frac{\partial}{\partial t}\left[e^{\left(\lambda^{2} D+\imath \lambda u\right) t} \widehat{C}(\lambda, t)\right]= & -\left[\left.D \frac{\partial C(x, t)}{\partial x}\right|_{x=0}-u C(0, t)\right] e^{\left(\lambda^{2} D+\imath \lambda u\right) t} \\
& -\imath D \lambda C(0, t) e^{\left(\lambda^{2} D+\imath \lambda u\right) t}
\end{aligned}
$$

Integrating in time, equation (A7) becomes

$$
\begin{aligned}
e^{\left(\lambda^{2} D+\imath \lambda u\right) t} \widehat{C}(\lambda, t)-\widehat{C}(\lambda, 0) & =-\int_{0}^{t}\left[\left.D \frac{\partial C(x, \tau)}{\partial x}\right|_{x=0}-u C(0, \tau)\right] e^{\left(\lambda^{2} D+\imath \lambda u\right) \tau} d \tau \\
& -\imath D \lambda \int_{0}^{t} C(0, \tau) e^{\left(\lambda^{2} D+\imath \lambda u\right) \tau} d \tau .
\end{aligned}
$$

Recalling that $C(0, t)=g_{o}(t)$, equation A8 can be written in the more compact form

$$
e^{\left(\lambda^{2} D+\imath \lambda u\right) t} \widehat{C}(\lambda, t)=\widehat{C}_{o}(\lambda)-\widehat{g}_{1}(\lambda, t)-\imath \lambda \widehat{g}_{o}(\lambda, t),
$$

with $\widehat{C}_{o}(\lambda), \widehat{g}_{1}(\lambda, t)$ and $\widehat{g}_{o}(\lambda, t)$ defined in equations 10$)-(12)$. Equation A9 is identical to the global relation $(9$ ).

\section{Appendix B}

Analyzing equation (25), it is clear that $\lambda=0$ is a removable singularity:

$$
\lim _{\lambda \rightarrow 0}\left[\frac{1-e^{-\left(\lambda^{2} D+\imath \lambda u\right) t}}{\lambda}\right]=\text { uut. }
$$




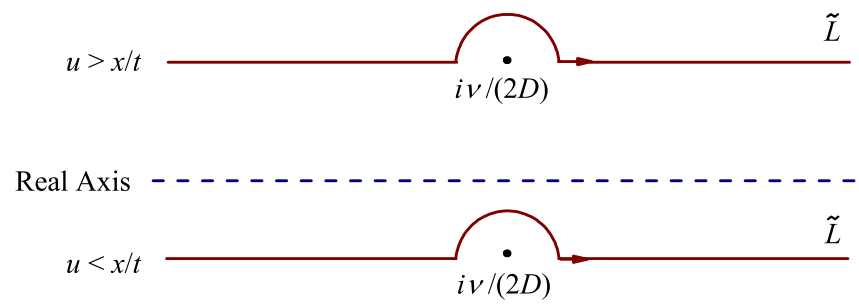

Figure 11: Mapping the singularity $\lambda=0$ to the point $\Lambda=\imath \nu /(2 D)$, with $\nu:=u-x / t$, in the $\Lambda$ complex plane with the new contour $\widetilde{L}$.

Thus, before we split 25) with the goal of obtaining a simpler expression, we first deform $\partial D^{+}$to go above $\lambda=0$ (i.e. bypassing the singularity). We call this new contour $L$.

In order to simplify the above solution $(25)$, we divide $C(x, t)$ into two terms

$$
C(x, t)=C_{1}(x)+C_{2}(x, t),
$$

with

$$
\begin{aligned}
C_{1}(x) & =-\frac{\imath C_{i}}{2 \pi} I_{1}(x) ; \\
C_{2}(x, t) & =\frac{\imath C_{i}}{2 \pi} I_{2}(x, t),
\end{aligned}
$$

where $I_{1}(x)$ and $I_{2}(x, t)$ are given by

$$
\begin{aligned}
I_{1}(x) & =\int_{L}\left(\frac{2 D \lambda+\imath u}{\imath u+D \lambda}\right) \frac{e^{\imath \lambda x}}{\lambda} d \lambda, \\
\text { and } \quad I_{2}(x, t) & =\int_{L}\left(\frac{2 D \lambda+\imath u}{\imath u+D \lambda}\right) \frac{e^{\imath \lambda x}}{\lambda} e^{-\left(\lambda^{2} D+\imath \lambda u\right) t} d \lambda .
\end{aligned}
$$

We can use Cauchy's theorem in the domain above $L$, where neither $\lambda=0$ nor $\lambda=-u \imath / D$ are in this domain. As a consequence, we find $I_{1}(x)=0$. In other words, $C_{1}(x)=0$.

In order to simplify $I_{2}$ and compute $C_{2}(x, t)$, we observe that the argument of the exponential term in $(\mathrm{B} 5)$ can be re-written as follows: 


$$
\begin{aligned}
-\left(\lambda^{2} D+\imath \lambda u\right) t+\imath \lambda x & =-t D\left[\lambda^{2}+\frac{\imath \lambda}{D}\left(u-\frac{x}{t}\right)\right] \\
& =-t D\left[\lambda^{2}+\frac{\imath \lambda}{D} \nu\right] \\
& =-t D\left[\lambda^{2}+\frac{\imath \lambda}{D} \nu-\left(\frac{\nu}{2 D}\right)^{2}+\left(\frac{\nu}{2 D}\right)^{2}\right] \\
& =-t D\left(\lambda+\imath \frac{\nu}{2 D}\right)^{2}-t \frac{\nu^{2}}{4 D},
\end{aligned}
$$

with $\nu:=u-x / t$. Therefore, $C_{2}(x, t)$, see equation $(\overline{\mathrm{B} 3})$, becomes

$$
I_{2}(x, t)=e^{-t \frac{\nu^{2}}{4 D}} \int_{L}\left(\frac{2 D \lambda+\imath u}{D \lambda+\imath u}\right) \frac{e^{-t D\left[\lambda+\imath \frac{\nu}{2 D}\right]^{2}}}{\lambda} d \lambda .
$$

If we employ the change of variables $\Lambda=\lambda+\imath \nu /(2 D)$, the expression for $I_{2}$ can be re-written in the form

$$
I_{2}(x, t)=e^{-t \frac{\nu^{2}}{4 D}} \int_{\widetilde{L}}\left[\frac{2 D \Lambda+\imath(u-\nu)}{D \Lambda+\imath\left(u-\frac{\nu}{2}\right)}\right] \frac{e^{-t D \Lambda^{2}}}{\Lambda-\imath \frac{\nu}{2 D}} d \Lambda,
$$

where the contour $\widetilde{L}$ is depicted in Figure 11 .

Comparing equations (B7) and (B8), it follows that the point $\lambda=0$ is mapped to the point $\Lambda=\imath \nu /(2 D)$. Thus, we need to distinguish the following two cases: (1) $u>x / t$, and (2) $u<x / t$. These two cases are depicted in Figure 11 where the new contour $\widetilde{L}$ is either above the real axis of the $\Lambda$ plane if $u>x / t$, or below if $u<x / t$. In the second case, we can deform the contour

$355 \widetilde{L}$ to the real axis; however, in the first case we also obtain a pole contribution from $\Lambda=\imath \nu /(2 D)$.

$$
I_{2}(x, t)=\left\{\begin{array}{cc}
e^{-t \frac{\nu^{2}}{4 D}} \widetilde{I}_{2}(x, t) \quad, \quad \text { if } u<\frac{x}{t} \\
e^{-t \frac{\nu^{2}}{4 D} \widetilde{I}_{2}(x, t)-2 \imath \pi}, \quad \text { if } u>\frac{x}{t}
\end{array}\right.
$$

with

$$
\widetilde{I}_{2}(x, t) \doteqdot \int_{-\infty}^{+\infty}\left[\frac{2 D \Lambda+\imath(u-\nu)}{D \Lambda+\imath\left(u-\frac{\nu}{2}\right)}\right] \frac{e^{-t D \Lambda^{2}}}{\Lambda-\imath \frac{\nu}{2 D}} d \Lambda .
$$


Note that $\Lambda=-\imath(u-\nu / 2) / D=-\imath(u+x / t) /(2 D)$ never contributes to $I_{2}$ since it is below the real axis.

Making use of equations $(\mathrm{B} 3),(\mathrm{B} 9),(\overline{\mathrm{B} 10})$ and substituting into $(\mathrm{B} 1)$ yields

$$
C(x, t)=\left\{\begin{array}{cc}
\frac{\imath C_{i}}{2 \pi} e^{-t \frac{\nu^{2}}{4 D}} \int_{-\infty}^{+\infty}\left[\frac{2 D \Lambda+\imath(u-\nu)}{D \Lambda+\imath\left(u-\frac{\nu}{2}\right)}\right] \frac{e^{-t D \Lambda^{2}}}{\Lambda-\imath \frac{\nu}{2 D}} d \Lambda \quad, \quad \text { if } u<\frac{x}{t} \\
\frac{\imath C_{i}}{2 \pi}\left[-2 \imath \pi+e^{-t \frac{\nu^{2}}{4 D}} \int_{-\infty}^{+\infty}\left[\frac{2 D \Lambda+\imath(u-\nu)}{D \Lambda+\imath\left(u-\frac{\nu}{2}\right)}\right] \frac{e^{-t D \Lambda^{2}}}{\Lambda-\imath \frac{\nu}{2 D}} d \Lambda\right], & \text { if } u>\frac{x}{t} .
\end{array}\right.
$$

In summary, equation (25) can be re-written in the form (B11). To make this suitable for quadrature, we let $\tau=\sqrt{t D} \Lambda$ and rewrite the integral in the dimensionless form

$$
\widetilde{I}_{2}(x, t)=\int_{-\infty}^{+\infty}\left[\frac{2 \tau+\imath \frac{\xi}{\sqrt{\eta}}}{\tau+\imath \frac{\sqrt{\eta}+\frac{\xi}{\sqrt{\eta}}}{2}}\right] \frac{e^{-\tau^{2}}}{\tau-\imath \frac{\sqrt{\eta}-\frac{\xi}{\sqrt{\eta}}}{2}} d \tau .
$$

For $\eta$ close to $\xi$, we deform this contour into the complex plane to avoid the pole.

\section{Acknowledgements}

F.P.J. de B. acknowledges the support from the National Science Foundation grant no. EAR-1654009. M.J.C. is supported by EPSRC grant no. EP/L016516/1. A.S.F. is supported by EPSRC, UK, via the senior fellowship. The numerical code used to obtain the results displayed in all figures is also available from M.J.C. upon request.

\section{References}

[1] G. Taylor, Dispersion of soluble matter in solvent flowing slowly through a tube, Proc. R. Soc. Lond. A 219 (1137) (1953) 186-203.

[2] A. Ogata, R. Banks, A solution of the differential equation of longitudinal dispersion in porous media: fluid movement in earth materials, Vol. 411, US Government Printing Office, 1961. 
[3] M. van Genuchten, W. Alves, Analytical solutions of the one-dimensional convective-dispersive solute transport equation, Technical Bulletins (157268).

[4] A. Kreft, A. Zuber, On the physical meaning of the dispersion equation and its solutions for different initial and boundary conditions, Chem Eng Sci. 33 (11) (1978) 1471-1480.

[5] T.-C. Lee, Applied mathematics in hydrogeology, CRC Press, 1998.

[6] J. S. P. Guerrero, L. C. G. Pimentel, T. H. Skaggs, M. T. Van Genuchten, Analytical solution of the advection-diffusion transport equation using a change-of-variable and integral transform technique, International Journal $385 \quad$ of Heat and Mass Transfer 52 (13-14) (2009) 3297-3304.

[7] J. S. P. Guerrero, E. M. Pontedeiro, M. T. van Genuchten, T. H. Skaggs, Analytical solutions of the one-dimensional advection-dispersion solute transport equation subject to time-dependent boundary conditions, Chemical Engineering Journal 221 (2013) 487-491.

[8] D. Tang, E. Frind, E. Sudicky, Contaminant transport in fractured porous media: analytical solution for a single fracture, Water Resour. Res. 17 (3) (1981) 555-564.

[9] E. Sudicky, E. Frind, Contaminant transport in fractured porous media: Analytical solutions for a system of parallel fractures, Water Resour. Res. 18 (6) (1982) 1634-1642.

[10] D. Roubinet, J.-R. Dreuzy, D. Tartakovsky, Semi-analytical solutions for solute transport and exchange in fractured porous media, Water Resour. Res. 48 (1).

[11] G. Dagan, Theory of solute transport by groundwater, Annu. Rev. Fluid Mech. 19 (1) (1987) 183-213. 
[12] D. Barry, G. Sposito, Analytical solution of a convection-dispersion model with time-dependent transport coefficients, Water Resour. Res. 25 (12) (1989) 2407-2416.

[13] H. Fischer, J. List, C. Koh, J. Imberger, N. Brooks, Mixing in inland and coastal waters, Academic Press, 1979.

[14] F. P. J. de Barros, W. B. Mills, R. M. Cotta, Integral transform solution of a two-dimensional model for contaminant dispersion in rivers and channels with spatially variable coefficients, Environ Model Softw 21 (5) (2006) 699-709.

[15] F. P. J. de Barros, R. M. Cotta, Integral transforms for three-dimensional steady turbulent dispersion in rivers and channels, Appl Math Model. 31 (12) (2007) 2719-2732.

[16] S. Rubol, I. Battiato, F. P. J. de Barros, Vertical dispersion in vegetated shear flows, Water Resources Research 52 (10) (2016) 8066-8080.

415 [17] V. Ganti, M. M. Meerschaert, E. Foufoula-Georgiou, E. Viparelli, G. Parker, Normal and anomalous diffusion of gravel tracer particles in rivers, J. Geophys. Res.: Earth Surface 115 (F2).

[18] H. Carslaw, J. Jaeger, Conduction of heat in solids, Oxford, England, 1959.

[19] J. Crank, The mathematics of diffusion, Oxford university press, 1979.

${ }_{420}[20]$ N. Ozisik, Heat conduction, John Wiley \& Sons, 1993.

[21] A. S. Fokas, A unified approach to boundary value problems, SIAM, 2008.

[22] B. Deconinck, B. Pelloni, N. Sheils, Non-steady-state heat conduction in composite walls, Proc. R. Soc. A 470 (2165) (2014) 20130605.

[23] N. E. Sheils, B. Deconinck, Heat conduction on the ring: Interface problems with periodic boundary conditions, Applied Mathematics Letters 37 (2014) 107-111. 
[24] N. Flyer, A. S. Fokas, A hybrid analytical-numerical method for solving evolution partial differential equations. i. the half-line, in: Proc. R. Soc. Lond. A, Vol. 464, The Royal Society, 2008, pp. 1823-1849.

[25] D. Mantzavinos, A. S. Fokas, The unified method for the heat equation: I. non-separable boundary conditions and non-local constraints in one dimension, European Journal of Applied Mathematics 24 (6) (2013) 857-886.

[26] E. Kesici, B. Pelloni, T. Pryer, D. Smith, A numerical implementation of the unified fokas transform for evolution problems on a finite interval, European Journal of Applied Mathematics 29 (3) (2018) 543-567.

[27] M. Asvestas, A. G. Sifalakis, E. P. Papadopoulou, Y. G. Saridakis, Fokas method for a multi-domain linear reaction-diffusion equation with discontinuous diffusivity, in: Journal of Physics: Conference Series, Vol. 490, IOP Publishing, 2014, p. 012143.

[28] P. D. Miller, D. A. Smith, The diffusion equation with nonlocal data, arXiv preprint arXiv:1708.00972.

[29] A. Fokas, A unified transform method for solving linear and certain nonlinear pdes, in: Proc. R. Soc. Lond. A, Vol. 453, The Royal Society, 1997, pp. 1411-1443.

[30] J. V. Uspensky, On the convergence of quadrature formulas related to an infinite interval, Transactions of the American Mathematical Society 30 (3) (1928) 542-559.

[31] D. Lubinsky, Geometric convergence of Lagrangian interpolation and numerical integration rules over unbounded contours and intervals, Journal of approximation theory 39 (4) (1983) 338-360.

[32] G. Mastroianni, G. Monegato, Error estimates for Gauss-Laguerre and Gauss-Hermite quadrature formulas, in: Approximation and Computation: A Festschrift in Honor of Walter Gautschi, Springer, 1994, pp. 421-434. 
[33] A. Glaser, X. Liu, V. Rokhlin, A fast algorithm for the calculation of the roots of special functions, SIAM Journal on Scientific Computing 29 (4) (2007) 1420-1438.

[34] A. Townsend, T. Trogdon, S. Olver, Fast computation of Gauss quadrature nodes and weights on the whole real line, IMA Journal of Numerical Analysis 36 (1) (2015) 337-358.

[35] D. Huybrechs, P. Opsomer, Construction and implementation of asymptotic expansions for Laguerre-type orthogonal polynomials, IMA Journal of Numerical Analysis 38 (3) (2017) 1085-1118.

[36] R. D. Skeel, M. Berzins, A method for the spatial discretization of parabolic equations in one space variable, SIAM journal on scientific and statistical computing 11 (1) (1990) 1-32.

[37] L. Sphaier, R. Cotta, C. Naveira-Cotta, J. Quaresma, The unit algorithm for solving one-dimensional convection-diffusion problems via integral transforms, International Communications in Heat and Mass Transfer 38 (5) (2011) 565-571.

[38] B. Pelloni, Well-posed boundary value problems for linear evolution equations on a finite interval, in: Mathematical Proceedings of the Cambridge Philosophical Society, Vol. 136, Cambridge University Press, 2004, pp. 361-382.

${ }_{475}[39]$ M. J. Colbrook, Z. I. Botev, K. Kuritz, S. MacNamara, Kernel density estimation with linked boundary conditions, arXiv preprint arXiv:1809.07735.

[40] E. A. Spence, Boundary value problems for linear elliptic PDEs, Ph.D. thesis, University of Cambridge (2011).

[41] K. Kalimeris, Initial and boundary value problems in two and three dimensions, Ph.D. thesis, University of Cambridge (2010). 